\title{
O USO DE AMBIENTES VIRTUAIS DE COLABORAÇÃO COMO FERRAMENTA PARA DESENVOLVIMENTO DE MÉTODO DE ENTURMAÇÃO
}

\author{
Waldir Siqueira Moura ${ }^{1}$, Mônica Ferreira da Silva ${ }^{1}$, Jonice de Oliveira Sampaio ${ }^{1}$ \\ ${ }^{1}$ Programa de Pós-graduação em Informática (PPGI) - Universidade Federal do Rio de \\ Janeiro (UFRJ) - Rio de Janeiro - RJ - Brasil \\ waldirsmeufrj.br; monica.silva@ppgi.ufrj.br, joniceddcc.ufrj.br
}

\begin{abstract}
This paper presents the first data analysis and discussions about the use of virtual environments to construct the workgroup method, considering the native instances of each student. We are based on the social network analysis of a sample of 40 students from a private network where a strong correlation between poor performance and their interrelations was found. From this reality, we understand that it is necessary to create a methodology of clutter that allows a more efficient grouping model by tangenting the native capacities among the students. To operationalize the creation of this methodology was used strategy based on Crowdsourcing from the Microsoft 365 platform.
\end{abstract}

Resumo. Este artigo apresenta as primeiras análises de dados e discussões sobreo uso de ambientes virtuais para a construção do método de grupos de trabalhos, considerando as instâncias nativas de cada estudante. Temos como fundamentoa análise da rede social de uma amostra de 40 estudantes de uma rede particularonde foi constatado uma correlação forte entre baixo desempenho e suas inter- relações. Partindo desta realidade, compreendemos que faz-se necessária a criação de uma metodologia de enturmação que permita um modelo de agrupamento mais eficiente, tangenciando as capacidades nativas entre osalunos. Para operacionalizar a criação desta metodologia utilizou-se estratégiabaseada em Crowdsourcing a partir da plataforma Microsoft 365.

\section{Introdução}

A educação formal é parte fundamental da sociedade e, portanto, é culturalmente valiosa. Desde o início do século XX a preocupação em compreender o motivo pelo qual algumas crianças e jovens têm dificuldades de aprendizagem cresceu consideravelmente como destaca Siqueira [2010].

As dificuldades de aprendizagem são problemas associados a muitas etiologias e, independentemente delas, esses fatores tem como resultado a baixa autoestima, desmotivação e problemas de relacionamentos, tanto no âmbito pessoal quanto familiar. Justifica-se assim a importância de utilizar um processo para entendimento e aprimoramento dos processos de ensino- aprendizagem. Para tanto, esta pesquisa contemplou o uso de ambientes virtuais de colaboração para o processo de mineração de dados.

Para que possa ser delineada uma proposta de método de trabalho colaborativo a ser aplicado dentro da sala de aula a partir do conhecimento resultante do processo de mineração desenvolvido através de ambientes virtuais de colaboração, optou-se pela utilização de uma 
ferramenta da plataforma da Microsoft $365^{1}$, o SharePoint. Com essa ferramenta criouse umambiente colaborativo que integrou 160 professores das cidades de Sorocaba/SP, Santo André/SP, Tremembé/SP, Lorena/SP e Rio de Janeiro/RJ.

Como este artigo tem o objetivo de relatar a pesquisa ainda em seu processo de desenvolvimento utilizamos apenas a análise feita com este pequeno grupo de alunos em apenas uma unidade escolar - que foi feita após a elaboração da mesma com um grupo controle, que permitiu identificar as dificuldades que deveriam ser sanadas para a pesquisa final, no entanto destacamos que o objetivo é o de desenvolver a mesma em outras unidades escolares.

A troca de experiências nos trabalhos dentro de sala de aula pode ser, além de enriquecedora, um ambiente propício para o compartilhamento de conhecimentos e visões multifacetadas sobre o mesmo assunto, como expõe Gessinger [2008]; isto porque asatividades em grupo são meios propícios para o desenvolvimento de cada estudante, onde através das interações e colaborações, é possível desenvolver o tangenciamento ${ }^{2}$ de capacidades nativas, tais como somestesia ${ }^{3}$ e psiquismo ${ }^{4}$.

Os trabalhos em grupos dentro do ambiente escolar são práticas fundamentais para odesenvolvimento coletivo, como apresentado por Cohen [2017]. Embora este tipo de enturmação seja necessária para o desenvolvimento integral dos estudantes, não é uma atividade trivial; há aspectos e situações que podem interferir no resultado desta prática. A investigação da enturmação é o foco deste estudo, que privilegiou o uso de ambientes virtuais de colaboração para o processo de mineração de dados para fundamentara construção de um método para enturmação dentro de sala de aula. Espera-se, com esta investigação, levantar fundamentação teórica-prática com subsídios para a constatação dos meios relevantes e essenciais para um processo de enturmação mais eficiente.

O método proposto fundamenta-se na análise da mineração de dados que foi desenvolvida - em ambiente virtual de colaboração - pelos professores da Rede de Ensino em questão. Com base nos relatos, experiências e dados obtidos foi possível identificar padrões de repetição e, com isso, realizar a Extração Automática de Termos (EAT) - detecção de padrões e formação morfossintáticos dos termos tais como "substantivo-adjetivo" e "substantivo- preposição-substantivo". Segundo Lucca [2006], a partir destes padrões é possível extrair os contextos definitórios do discurso, obtendo-se assim uma taxonomia semântica das suas definições.

Segundo Lucca [2006], os contextos definitórios dos padrões de repetição aparecem, geralmente, em três circunstâncias: I. citação de um termo reservado; II. na utilização de umtermo novo ou pouco utilizado na comunidade a que se destina a pesquisa e III. quando é utilizado um termo que só possui caracterização em inglês (neste caso pode ocorrer da comunidade tentar encontrar um outro termo equivalente em português que, muitas vezes, pode resultar em um neologismo, e neste caso - como

\footnotetext{
${ }^{1}$ Pacote de aplicativos para escritório que contém programas como: processador de texto, planilha de cálculo, bancode dados, apresentação gráfica, cliente de e-mails, entre outros.

${ }^{2}$ É a articulação das instâncias somestésica e psíquica.

${ }^{3}$ Agrupa funções corporais correlacionadas às atividades chamadas inferiores.

${ }^{4}$ Agrupa competências para atividades chamadas superiores.
} 
esclarece Lucca [2006] - o neologismogeralmente vem acompanhado de sua definição, o que é chamado de contexto definitório ouexplicativo).

Este artigo visa apresentar as primeiras discussões sobre a análise da mineração dos dados elaborada em ambiente virtual de colaboração. As discussões foram realizadas atravésde um processo de Crowdsourcing realizado com o corpo docente. $\mathrm{O}$ objetivo do Crowdsourcing foi solicitar a contribuição da comunidade docente online para a elaboração de um método para otimizar o processo de enturmação e criação de grupos de trabalhos em sala de aula.

Esta atividade possibilitou uma melhor compreensão acerca do fenômeno dos contextos definitórios através da imersão na análise de Gêneros de Discurso ${ }^{5}$, que é fundamental para o reconhecimento do padrão de uso de palavras pertencentes a léxicos reservados. Para tanto, efetuou-se, manualmente, a detecção dos contextos definitórios com base nas experiências e propostas dadas pelos professores no SharePoint.

\section{Problema}

Os problemas fundamentais que motivaram esta pesquisa foram o baixo desempenho dos estudantes e a forma com que as enturmações acontecem dentro das salas de aula. Acredita-se que a enturmação pode propiciar a exclusão de alguns estudantes e grupos fechados, possibilitando a formação de grupos interesseiros com foco em nota, o que pode não favorecer o desenvolvimento da turma como um todo. É possível, pois, utilizar o processo de mineração textual para embasar estratégias de intervenção para otimização dos processos de enturmação e consequentemente, do ensinoaprendizagem?

Destacamos aqui que nossa pesquisa se baseia na análise do que chamamos de redes sociais reais, ou seja, de redes de relacionamentos presenciais - a pesquisa se desenvolveu anteriormente ao início da pandemia - sendo que consideramos as plataformas como Facebook, Twitter, Instagram e etc como mídias sociais e não redes sociais.

\section{Proposta de intervenção: o uso de ambientes virtuais como estratégia para a elaboração de um método}

Após o desenvolvimento das atividades conforme mostra a Figura 1, concluiu-se que, para a solução dos problemas levantados, a melhor proposta para uma solução seria ouvir, dos próprios professores que estão nas salas de aula diariamente quais os métodos mais utilizados para atividades em grupo.

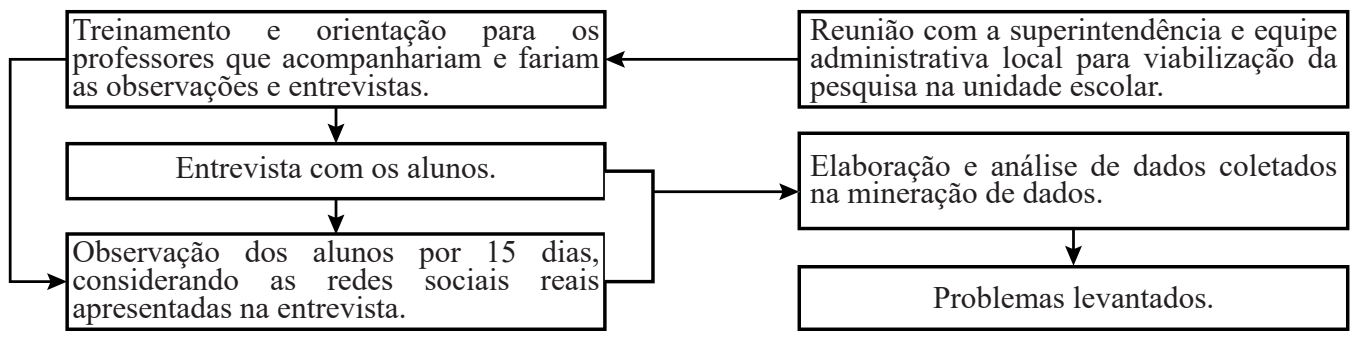

Figura 1. Apresentação gráfica da organização das etapas de trabalho

\footnotetext{
${ }^{5}$ Cada enunciado particular é individual, mas cada campo de utilização da língua elabora seus tipos relativamente estáveis de enunciados, os quais denominamos gêneros do discurso. Bakhtin, [2015]
} 
Como a rede de ensino possui seis Colégios localizados em dois estados diferentes e em cidades distantes, foi adotado o uso de um ambiente virtual de colaboração de modo que o trabalho poderia ser realizado de forma colaborativa com economia de tempo e deslocamento. Sendo a plataforma um meio definido como o uso da inteligência coletiva para obter resultados inovadores, refinados, inteligentes e mais precisos, este modelo de produção mostrou-se o mais adequado ao objetivo de uso do conhecimento descoberto na Mineração para tomada de decisão no contexto desta pesquisa.

A seleção da plataforma virtual considerou o ambiente computacional já utilizado pela amostra de docentes. Como benefício do aproveitamento do ambiente, citase que pelo fato de os professores já utilizarem-no, não houve necessidade de treinamento. A plataforma comum a todos os Colégios é o Microsoft 365, que conta com o Microsoft SharePoint, uma ferramenta que possibilita a criação e gestão de ambientes colaborativos. Esta ferramenta foi utilizada para promover a colaboração e a distribuição de tarefas entre grupos e equipes dentro da rede. Para tanto, criou-se uma comunidade fechada onde apenas o corpo docente tinha acesso; nessa comunidade, os docentes foram adicionados como colaboradores.

Foi solicitado aos professores que propusessem métodos que eles achassem adequados, experiências com métodos utilizados e outros tipos de tarefas, tais como comentar e aprimorar métodos propostos por outros professores. De 160 professores participantes, obteve-se 200 interações dentro do ambiente criado com a ferramenta. Após as contribuições feitas, as interações foram tabuladas para facilitar o processo de Extração Automática de Termos.

\section{População amostral}

Esta pesquisa foi realizada com uma amostra contendo 40 estudantes entre o primeiro e terceiro ano do Ensino Médio de uma Rede de Ensino particular que possui filiais no Rio deJaneiro e em São Paulo.

A pesquisa também contou com a participação de dois coordenadores pedagógicos para auxílio na coleta de dados iniciais e 160 docentes no processo de Crowdsourcing - queque foi desenvolvido com a utilização de um ambiente virtual de colaboração - distribuídos em 5 cidades localizadas nos 2 estados de abrangência da rede.

\section{Processo de mineração de dados}

O trabalho inicial constituiu na mineração de dados dos estudantes dentro do ambiente educacional. Para tanto, utilizou-se como instrumento de pesquisa a entrevista. A entrevista realizada compunha-se de 14 perguntas fechadas e objetivas e, por exemplo, cada aluno - em particular - poderia indicar os componentes de sua rede social livremente, sem número definido, esta pergunta foi feita em dois aspectos sendo o primeiro os amigos que compunham sua rede de amigos pessoal e depois a rede de amigos que tinha para realização de trabalhos escolares e avaliações em duplas, e teve como objetivo principallevantar dados referentes às redes sociais dos estudantes, bem como suas preferências e informações sobre participação em atividades extracurriculares.

Esta atividade resultou na construção de grafos e no mapeamento das redes sociais dos estudantes. A etapa posterior teve como objetivo verificar e triangular a entrevista, e teve como instrumento de pesquisa a observação, sendo que os professores 
tiveram acesso as respostas das entrevistas e uma formação antes de darem início a triangularização. Para tanto, alguns professores, previamente instruídos, observaram os comportamentos dos alunos durante o período de 15 dias, dentro do ambiente educacional, a fim de constatar se as redes sociais apresentadas na entrevista correspondiam à realidade no quotidiano dos estudantes.

Com o término da etapa de observação, obteve-se novos grafos de mapeamento das redes sociais dos estudantes, conforme a Figura 2. Como apresentado no canto superior esquerdo da Figura 2, existem estudantes que, embora sejam da primeira série do Ensino Médio (demarcados com a cor laranja) não interagem com nenhum outro estudante de sua série; no entanto, interagem com alunos da segunda série do Ensino Médio. A análise dos dados retornou que estes alunos apresentam as médias mais baixas da turma. Outro fato interessante que merece destaque nesta mineração de dados é a forma comque as inter-relações se dão; com base neste grafo, é possível considerar pelo menos três tiposde interações distintas, sendo elas: amizade unilateral (quando apenas um estudante considerao outro amigo mas não é recíproco), amizade bilateral (quando os estudantes se consideram amigos mutuamente) e amizade por interesse (quando um estudante considera o outro como amigo apenas na realização de trabalhos); este último tipo de interação é representado pelassetas de cor rosa.
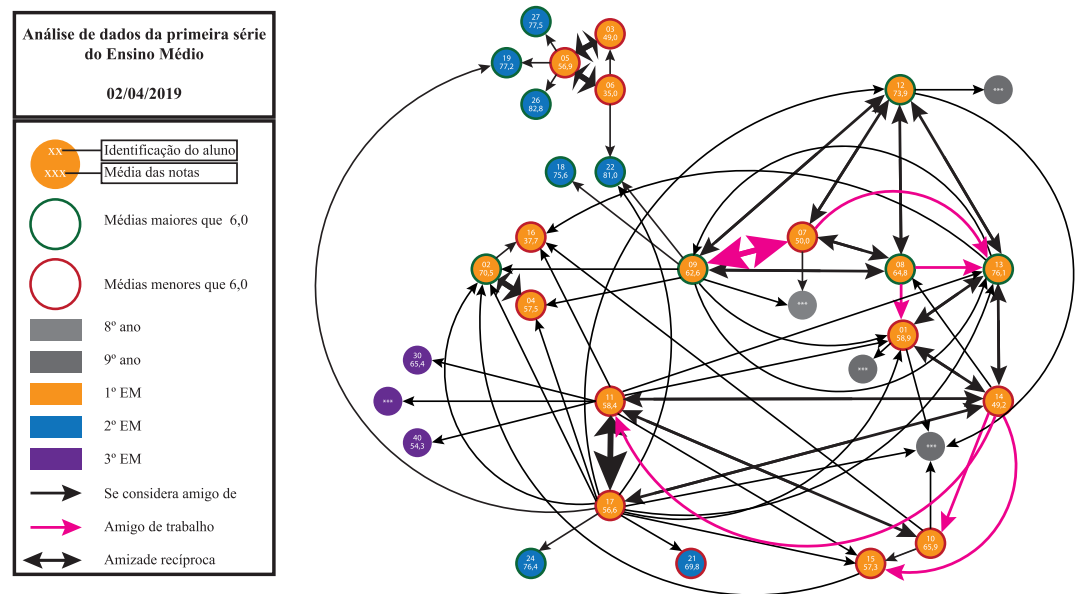

\section{Figura 2. Análise detalhada das Redes Sociais dos estudantes da primeira série do Ensino Médio}

Um fator interessante que advém destes três tipos de amizades é que há alguns alunos que são focos apenas para trabalhos, sendo eles os que possuem maior média da sala, o que pode reforçar a análise de que há, de fato, um envolvimento por interesse. Com essas informações, acreditou-se que quanto maior o número de entrada (número de amigos), maior seria a média do estudante. Para verificar e comprovar esta afirmação, utilizou-se o Coeficientede Correlação de Pearson (CCP).

O CCP é um índice adimensional com valores situados entre $-1,0$ e 1,0 que reflete a intensidade de uma relação linear entre dois conjuntos de dados. Nesta análise este coeficiente assume apenas valor entre -1 e 1, sendo 1 uma relação perfeitamente positiva entre as duas variáveis e -1 uma correlação negativa perfeita entre as duas variáveis. Assim, se uma variável aumenta, a outra diminui. Desta forma, considera-se que $r=0$ significa que as duas variáveis não dependem linearmente uma da outra.

Para estabelecer, pois, uma correlação entre a métrica in-degree e a média de notas (que correspondem ao desempenho do estudante na disciplina) utilizou-se o CCP. 
A partir da análise do gráfico, observa-se que há uma correlação linear positiva entreo grau de entrada e as notas. Isso significa, portanto, que quanto maior o número do grau deentrada, maior será a nota do estudante, ou seja, quanto mais amigos o aluno tiver maior seráa sua chance de alcançar uma nota melhor. Considerando que o CCP na análise desta série éde 0.7936 , pode-se concluir que esta correlação é muito forte.
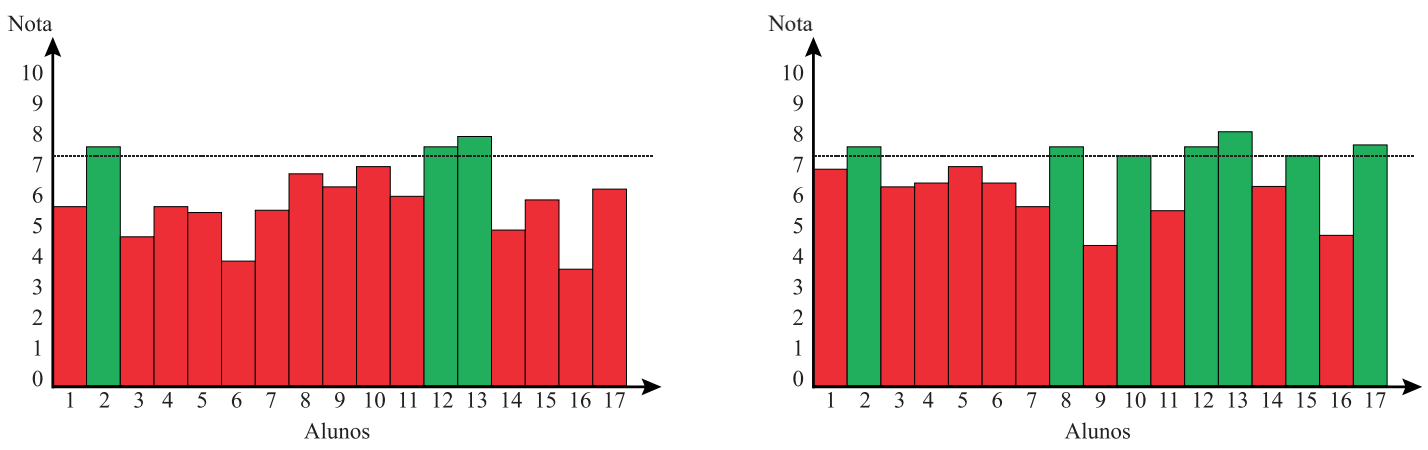

\section{Figura 3. Análise de desempenho dos estudantes da primeira Série do Ensino Médio referente ao primeiro e terceiro trimestre de 2019}

Outro conhecimento importante obtido a partir do processo de mineração de dados foi sobre o desempenho dos estudantes. Constatou-se que grande parte deles possui um baixo desempenho escolar, mesmo se tratando de uma rede particular, como é possível observar na Figura 2.

Como pode ser visto na tabela à esquerda da Figura 3, 82\% dos estudantes desta sala possuem desempenho abaixo da média no primeiro trimestre letivo e a tabela à direita da Figura 3 apresenta informações parciais sobre o desempenho desta mesma turma após a aplicação do método proposto em algumas atividades. Como pode ser percebido há uma melhora de $23 \%$ no rendimento dos alunos após uma intervenção programada no meio que considerou os dados levantados através da mineração dos dados.

Conforme observa-se na Figura 3, 59\% dos estudantes ainda apresentam média abaixo da exigida pelo Colégio. Note-se que a média de corte desta Rede de Ensino é 7,0. E a probabilidade de que esses dados sejam melhorados positivamente com a implementação do método é consideravelmente grande, principalmente seconsiderarmos os dados parciais coletados.

\section{Resultados}

Como resultado das atividades envolvendo Mineração de Dados, Extração Automática de Termos e uso de ambiente virtual colaborativo, foi elaborado um método unificado que deve trabalhar com competências e habilidades socioemocionais, mesmo que estas representem um desafio em sala de aula. Assim, foi possível elaborar atividades que garantirão condições de acesso, permanência, participação e aprendizado de modo a eliminar as barreiras criadas por esses estudantes e até mesmo pelos demais colegas a fim de promover a inclusão plena desses alunos, garantindo condições de igualdade a todos.

A partir da colaboração dos professores para a resolução do problema apresentado, elaborou-se a seguinte proposta de ação: Este método tem a avaliação como proposta de "triagem" para a percepção das capacidades de cada estudante para que assim possa se formar grupos de acordo com as habilidades diferentes, reforçando a importância e o reconhecimento de cada um. Selecionando o que cada um tem de potencial, acredita- 
se que é possível nivelar as inteligências de modo a elevar a autoestima e autoconfiança do estudante no seu ponto forte.

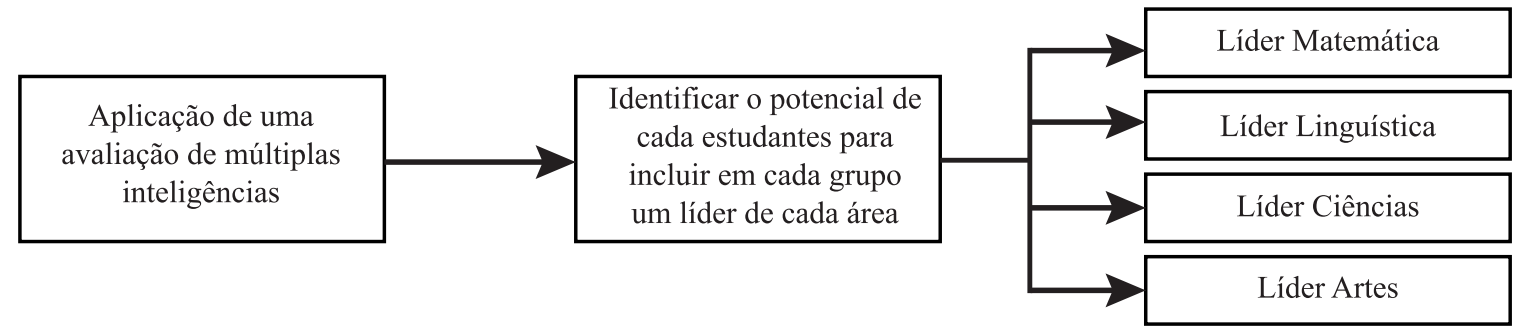

Figura 4. Apresentação gráfica do método desenvolvido

Ao elaborar estas atividades em grupo, deve-se procurar dar papéis específicos a cada participante, de forma a contribuir para que todos os membros possam, em algum momento, desempenhar a função de líder no grupo. Esses papéis devem variar em: mediador, monitor, o dono do tempo e o harmonizador, e todos devem transitar pelos diferentes papéis. Os grupos podem ser formados por afinidades ou interesses, conforme o resultado da avaliação, mas também devem contar com o direcionamento do professor de acordo com o objetivo a ser atingido. A implantação do método desenvolvido resultou em uma melhora considerável no desempenho acadêmico dos alunos que foram submetidos ao experimento.

\begin{tabular}{|c|c|c|c|c|c|c|c|c|c|c|}
\hline & $\begin{array}{l}\text { 苞 } \\
\text { Z }\end{array}$ & 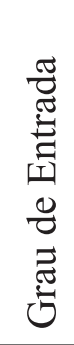 & 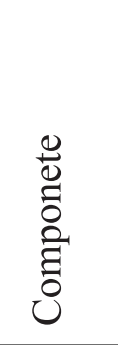 & 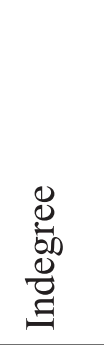 & 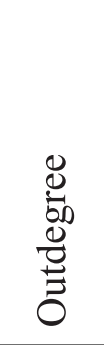 & 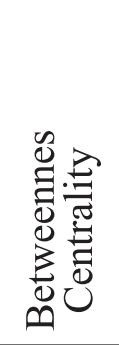 & 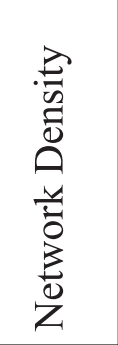 & 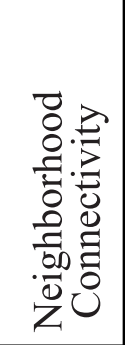 & 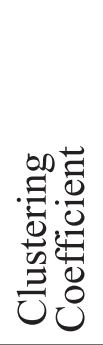 & 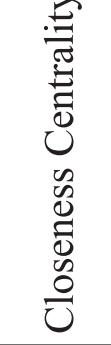 \\
\hline Antes & 5,47 & $\varnothing$ & G e $P$ & 3,17 & 3,23 & 0,579 & 0,145 & 5,010 & 0,52 & 0,548 \\
\hline Depois & 5,47 & $\varnothing$ & $\mathrm{G}$ & 5,88 & 6,17 & 0,404 & 0,276 & 7,705 & 0,42 & 0,546 \\
\hline
\end{tabular}

Figura 5. Quadro comparativo de média das métricas utilizadas

\section{Considerações finais e perspectivas futuras}

Esta pesquisa visou discutir os primeiros passos para a implantação do uso de uma plataforma de colaboração virtual para a solução de problemas escolares, especificamente na solução do problema de enturmação, investigado através da mineração de dados. A abordagem utilizada privilegiou a análise do discurso e das redes sociais para a análise de um grande fluxo de dados envolvendo o baixo desempenho escolar que é um problema atual da rede escolar em questão, e o problema de enturmação. Foi possível notar que o problema de agrupamento dentro de sala de aula é um problema constante que necessita de uma abordagem adequada para que possa acontecer de maneira produtiva e eficiente.

$\mathrm{O}$ processo de mineração textual embasou uma estratégia para otimizar a enturmação e consequentemente, o processo de ensino-aprendizagem. Para a elaboração da estratégia, utilizou-se o modelo de Crowdsourcing, com o objetivo de solicitar a 
contribuição da comunidade docente online para a elaboração do novo método de trabalho. Como proposta de trabalhos futuros, esta pesquisa visa conduzir a experimentação deste método na sala de aula que foi a base para a construção do método a fim de validar estes resultados parciais obtidos. Propõe-se também a elaboração de um engenho computacional que seja capaz de fazer a extração automática de termos e agrupálos de acordo com suas semânticas.

\section{Referências}

Boulic, Bakhtin, Mikhail. (2015). Os Gêneros do discurso. São Paulo: Editora 34. $5^{\text {a }}$ edição, p. 262. Cohen, E. G., Lotan, R. A. (2017) "Planejando o trabalho em grupo - estratégias para salas de aula heterogêneas", $3^{\mathrm{a}}$ ed, Penso, Instituto Sidarta.

Delbem, Edgar, sjr. (2014). Modelo computacional colaborativo para mensurar o desenvolvimento interrelacional em alunos do Ensino Médio. Dissertação (Mestrado em Informática) - Universidade Federal do Rio de Janeiro, Instituto de Matemática, Instituto Tércio Pacitti.

Gessinger, R.M. (2008). “Atividade em grupo, In: A gestão da aula universitária na PUCRS”, editado por Grillo, M.C., Freitas, A.L.S., Gessinger, R.M., Lima, V.M.R., EdiPUCRS, Porto Alegre. Figura 9: Quadro comparativo de média das métricas utilizadas.

Lucca, J. L. De. (2006). Identificação de padrões recorrentes no discurso técnico e científico para a extração automática a candidatos definitórios em Língua Portuguesa. In: Revista Intercâmbio, vol. XV. São Paulo: LAEL/PUC-SP.

Siqueira, Cláudia Machado; Gurge-Giannetti, Juliana. (2010). Poor school performance: an updated review In: Revista da Associação Médica Brasileira, Edited by Nadia Magnenat Thalmann and Daniel Thalmann, John Wiley \& Sons ltd., England.

Castro, Alberto; Menezes, Crediné. (2011). Aprendizagem colaborativa com suporte computacional. In Sistemas Colaborativos.

Pimentel, Mariano; Fuks, Hugo. (Orgs). Rio de Janeiro: Elsevier. P. 135. Colaço, V. De F. R. 2004. Processos interacionais e a construção de conhecimento e subjetividade de crianças. In Psicologia: Reflexão e Crítica, Porto Alegre, V. 17, N. 3, P. 333-340.

Damiani, Magda Floriana. (2008). Entendendo o trabalho colaborativo em educação e revelando seus benefícios. In Educar, Curitiba, N. 31, P. 213-230, Editora UFPR.

Gouvêa, Maria Teresa; Pimentel, Mariano; Santoro, Flavia Maria; Cappelli, Claudia. 2016. Externalização do conhecimento através de group storytelling: um estudo de caso em tutorial online. In XII Brazilian Symposium on Information Systems, Florianópolis, Sc, May 17-20.

Moura, Waldir Siqueira; Dias, Angélica Fonseca da Silva; França, Juliana Baptista dos Santos; Borges, Marcos Roberto da Silva; Oliveira, Jonice de Oliveira Sampaio. ColabSaber: Um framework de suporte pedagógico na construção colaborativa de saberes. In: WORKSHOP DE INFORMÁTICA NA ESCOLA, 26, 2020, Evento Online. Porto Alegre: Sociedade Brasileira de Computação, 2020, p. 6170. DOI: https://doi.org/10.5753/cbie.wie.2020.61.

Kotujansky, Silvio. (2009). Um modelo para a elaboração colaborativa de conteúdos didáticos digitais que utilizem a metáfora de histórias em quadrinhos e recursos hipermídia. Dissertação defendida na Universidade Federal de Santa Catarina como requisito para obtenção do grau de Mestre.

Mcsill, James. (2013). 5 Lições de storytelling: fatos, ficção e fantasia. 1. Ed. São Paulo: Dvs Editora. In Santos, Leonardo Schwertner Dos. Storytelling: O Poder da Narrativa Estratégica Dentro do Branding e Marketing. P. 5.

Neves, Sílvia Da Conceição. (2012). A História em quadrinhos como recurso didático em sala de aula. Trabalho de Conclusão de Curso de Artes Visuais, Habilitação em Licenciatura, do Departamento de Artes Visuais da Universidade de Brasília. Palmas. XAVIER JR., Joaquim Ferreira. "A PsicogenéticaDemarcando os processos da vida”. Tremembé: VespeR Editora. 2004. P. 337. 